\title{
Extraction Timing of Heavily Destructed Upper First Permanent Molars
}

\author{
Ahmad A. Rahhal \\ Department of Orthodontics, Faculty of Dentistry, Arab American University, Jenin, Palestine \\ Email: plorahhal@hotmail.com \\ Received 1 February 2014; revised 6 March 2014; accepted 14 March 2014 \\ Copyright (C) 2014 by author and Scientific Research Publishing Inc. \\ This work is licensed under the Creative Commons Attribution International License (CC BY). \\ http://creativecommons.org/licenses/by/4.0/ \\ (c) (i) Open Access
}

\section{Abstract}

The first permanent molar as the first permanent tooth in the mouth and with a general insufficient oral hygiene of the children, is commonly subject to significant compromise which may arise due to caries or endodontic complication, or from developmental anomalies such as hypoplasia. Compromised teeth with questionable prognosis may result in short- and long-term clinical dilemmas. The aim of this research is to highlight the factors that require careful consideration when a compromised UFPM is detected and the importance of timely UFPM extraction to ensure the mesial drift of the upper second permanent molar (USPM) to fulfill the space of the extracted UFPM without any orthodontic intervention. For this purpose 52 heavily destructed UFPMs were extracted at age of 10.5 years old, $44(84.6 \%)$ USPMs erupted exactly distal to the second premolar while only $7(13.4 \%)$ USPMs erupted $1 \mathrm{~mm}$ distal to the second premolar. Clinically it is recommended to extract the heavily destructed upper first molars at age of $\mathbf{1 0 . 5}$ years old to ensure the complete closure of the extraction space by the passive mesial drift of upper second permanent molar.

\section{Keywords}

First Permanent Molar (FPM); Upper First Permanent Molar (UFPM); Upper Second Permanent Molar (USPM); Heavily Destructed UFPM; Timing of Extraction; Extraction Space Closure; Passive Mesial Drift

\section{Introduction}

The first permanent molar (FPM) has been reported to be the most caries prone tooth in the permanent dentition. More than 50\% of children over the age of 11 years have some caries experience in this tooth [1]. In addition to this, the FPM has also been commonly found to be significantly hypoplastic, with approximately $6 \%$ of children having hypoplasia in one or more first permanent molars [2]. 
Orthodontic cases involving the extraction of the first permanent molars are usually more technically demanding compared with conventional premolar extraction or non-extraction treatment. However, it could be argued that many patients would benefit from extraction of compromised FPMs due to their questionable longterm prognosis. A decision to electively extract healthy premolar teeth for orthodontic purposes may not be justifiable in these cases.

There are many factors to consider before extracting any FPM. Cases involving extraction of FPMs are frequently associated with protracted orthodontic treatment and more complicated mechanics. However, appropriate timing of FPM extractions can greatly facilitate and simplify subsequent fixed appliance orthodontic treatment. As with any form of dental treatment, the cost-benefit perspective of FPM extraction and subsequent fixed appliance orthodontic treatment must be carefully evaluated.

The first permanent molar is rarely the tooth of choice for extraction prior to orthodontic treatment. However, there are various clinical situations in which extraction of FPMs should be considered [3]: extensively carious FPMs; hypoplastic FPMs; heavily restored FPMs where premolars are perfectly healthy; apical pathoses or endodontically treated FPMs; crowding at the distal aspect of the arches and third molars of reasonable form and in reasonable position; skeletally divergent malocclusions (dolichofacial vertical pattern; and anterior open bite malocclusion).

Extraction of FPMs before the age of 8 years has the potential to result in distal drifting, tilting and rotation of the adjacent unerupted second premolar tooth. This can occur if the second premolar is distally inclined and "escapes" from the bifurcation of the second primary molar roots. It will then lack the guidance normally provided by the FPM and can erupt distally into the FPM socket [4].

Timing of FPM removal is generally more critical in the lower arch. However, timing of FPM removal for the upper arch may also be important depending on the type of buccal segment relationship present.

The overall approach and timing of FPM extractions can vary with different occlusal relationships. It is imperative to assess the over jet, overbite and posterior occlusion when considering any FPM extraction [5].

In Angle CLI if a lower FPM is compromised, a balancing extraction in the lower arch and a compensating upper FPM extraction should be considered. This is particularly important for mixed dentition cases. If an upper FPM is compromised, a balancing extraction in the upper arch should be considered to prevent a midline shift if deemed to be necessary. However, a compensating FPM extraction in the lower arch is generally not required due to the inter cuspation between the lower first molar and the upper second permanent premolar [6].

In compromised FPM cases where significant intra-arch crowding exists and permanent tooth extractions are required for tooth alignment, the extraction of one or more compromised FPMs can certainly be justified, rather than removing sound premolar teeth. In general, orthodontic space closure of FPM extraction spaces is much easier in cases with significant intra-arch crowding.

In cases where the intra-arch crowding is minimal, the timing of compromised FPM removal is far more critical to avoid large residual spaces by maximizing the amount of mesial drift of the second permanent molar teeth. Closing large residual spaces in the absence of significant crowding has the potential to negatively affect soft tissue profile aesthetics through undesirable anterior segment retraction.

It is imperative to conduct a comprehensive clinical and radiographic examination to confirm the presence and condition of the remaining teeth. A panoramic film can determine the presence of any developing third molar teeth. The absence of third molar teeth does not necessarily contraindicate the extraction of compromised FPMs [5] [6].

The decision to remove compromised FPMs when the third molars are not present is very much case-dependent. Significantly compromised FPMs which would require extensive future restoration or likely extraction would seem reasonable to remove. On the other hand, maintenance of compromised FPMs with acceptable long-term prognosis may be warranted in cases where extraction space closure is determined to be undesirable or unpredictable. In cases where third molar teeth are present, space gained from the extraction of compromised FPMs can greatly increase the likelihood of, although not guarantee, successful eruption of these third molar teeth [7] [8].

\section{Timing of UFPM Extraction}

The USPM can erupt into a satisfactory contact point relationship with the upper second premolar if the USPM was unerupted at the time of the UFPM extraction. In particular, very favourable space closure can be achieved if the USPM is located above the cemento-enamel junction of the UFPM to be extracted. [5] [9] in our research 
we tried to predict the best time for the extraction of upper first molars despite the difference in sex between the patients, and the relation between the crown of the second molar and the cemntoenamel junction of the first molar which might cause some difference in the eruption time of the second molar teeth.

\section{Predicting the Extraction Time for the Research}

In our research the prediction of the extraction time as 10.5 years old was according to the following tooth physiologic movement principles [10] [11]:

1) When a tooth is extracted the neighboring teeth will drift to the extraction space.

2) The drift movement is more a tipping than bodily movement. Due to the long axis of the teeth the tipping movement in the lower arch is more than the upper arch.

3) The mesial drift movement of the unerupted teeth (still inside the bone) is very near to the bodily movement specially in the upper arch.

4) Due to the long axis of the teeth, the spontaneous closure of the extraction space in the upper arch is better than the lower arch.

5) The younger the patient is, the faster the eruption movement of the succedanea tooth.

6) If the deciduous tooth was extracted 2 years or more before the eruption time of the succedanea permanent tooth, the eruption time will delay, but if it was extracted 2 years or before the eruption time then the eruption of the succedanea tooth will accelerate.

Due to all the previous principles we believed that the extraction of the heavily destructed UFPM at age of 10.5 years old will accelerate the eruption and mesial drift of the USPM in a bodily movement to produce a complete or satisfactory extraction space closure without orthodontic intervention.

\section{Material and Method}

The patients were selected from the clinic of the Arab American University-Jenin through five years time. 52 heavily destructed UFPM tooth were followed up, and then extracted at age of 10.5 years old, all cases were Angle CLI, to prevent the over eruption of the lower first permanent molar. In the majority of the cases the upper third permanent molar was not missing.

\section{Results}

44 (84.6\%) USPM tooth erupted exactly distal to the upper second permanent premolar without any orthodontic intervention (Figures 1-5), while only 8 (15.4\%) USPM tooth erupted $1 \mathrm{~mm}$ distal to the upper second permanent premolar (Figures 6-8).

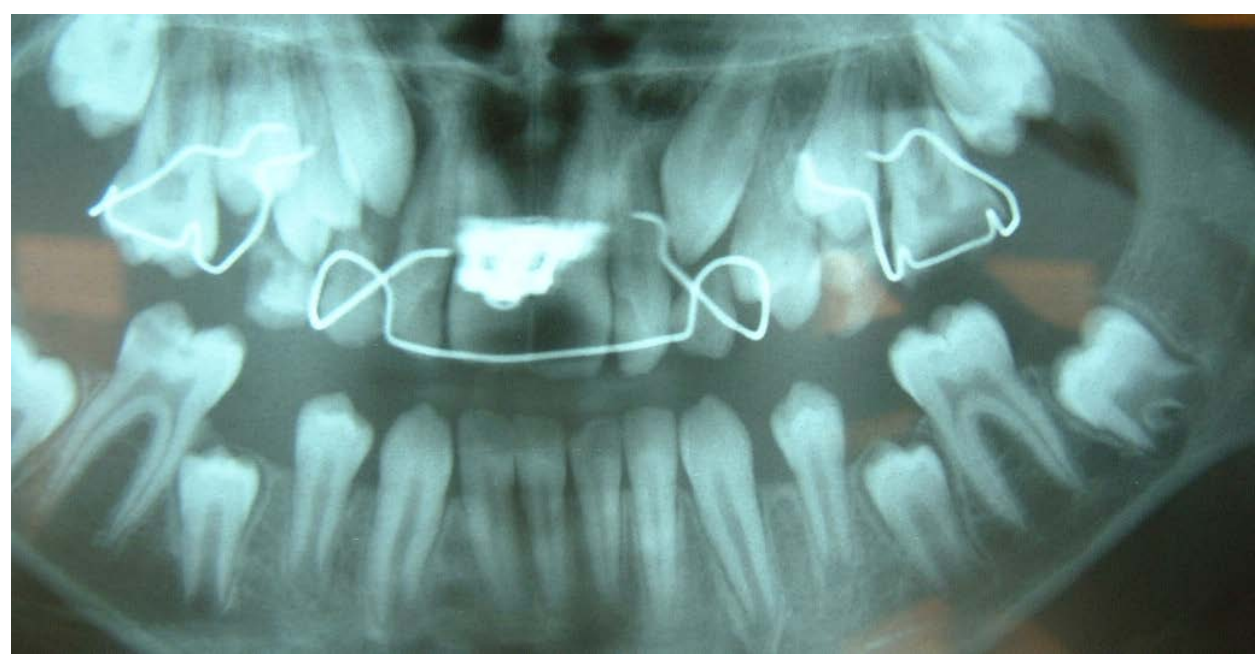

Figure 1. This panoramic was taken exactly before the extraction of the upper left first molar. Upper right first molar undergone RCT. The expansion appliance was removed and started with fixed orthodontic appliance to level the anterior teeth. 


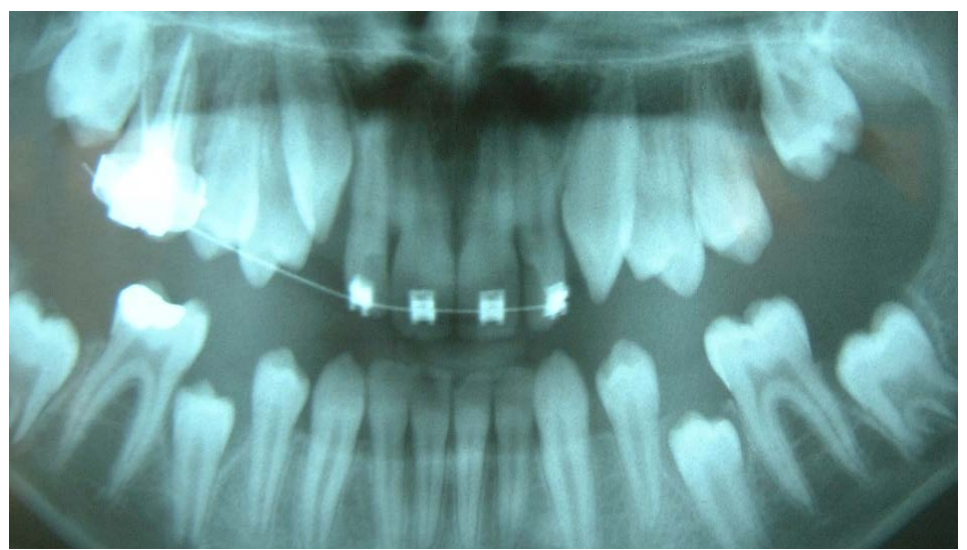

Figure 2. The same patient after 6 months of extraction of the upper left first permanent molar.

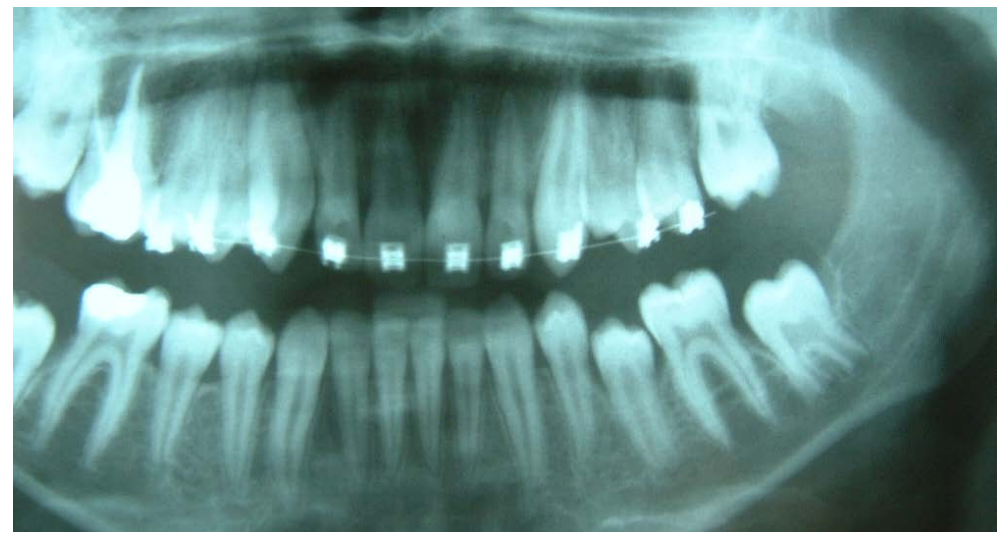

Figure 3. One year after the extraction of the upper left first molar.

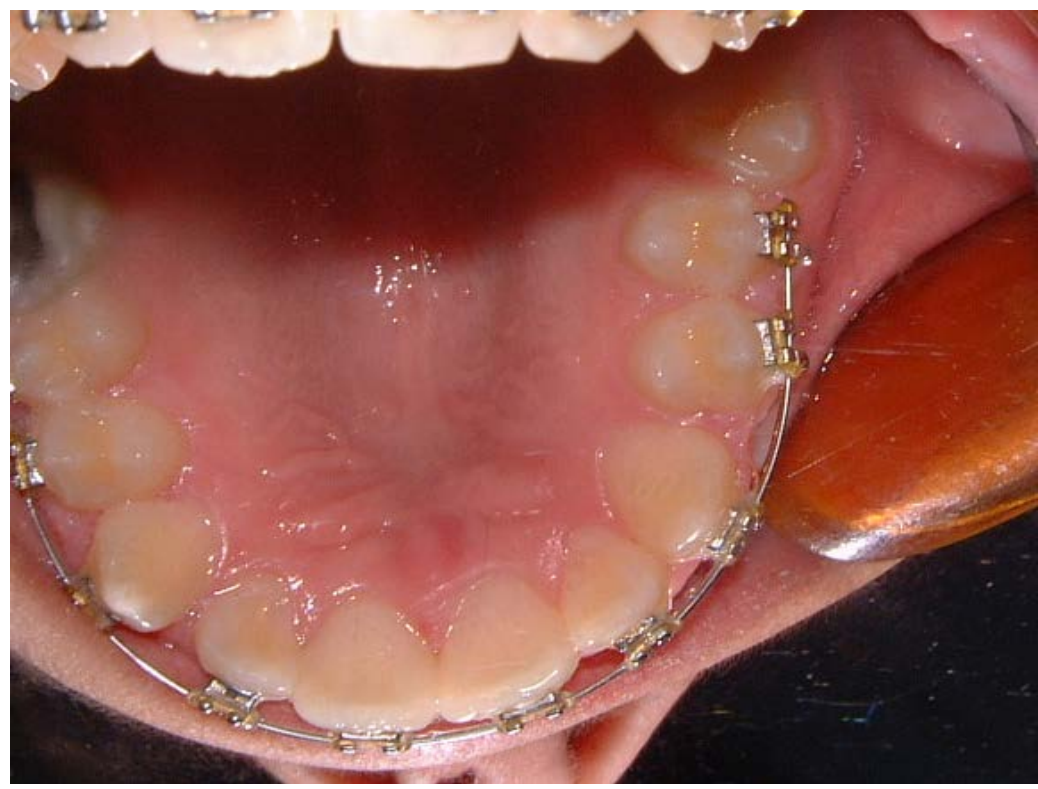

Figure 4. Intraoral photograph of the same patient one year after the extraction, the patient is 11.5 years old, the upper second molar erupted exactly distal to the first premolar. 


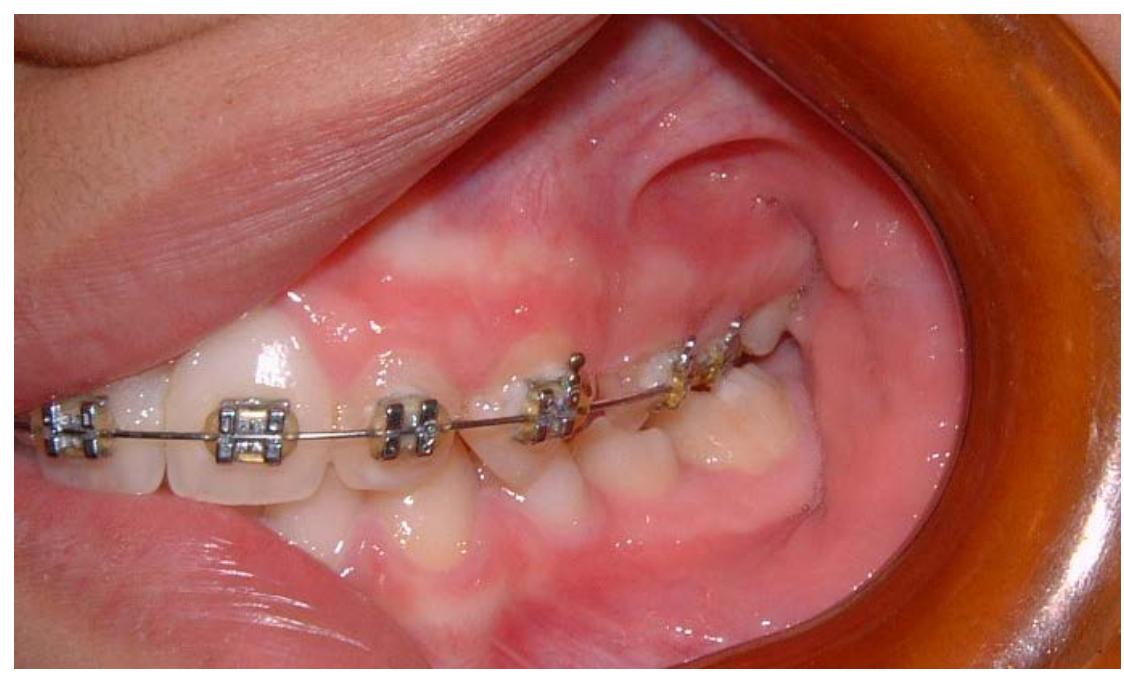

Figure 5. 16 months after the extraction, the upper second molar erupted in place and without any cross bite or tilting.

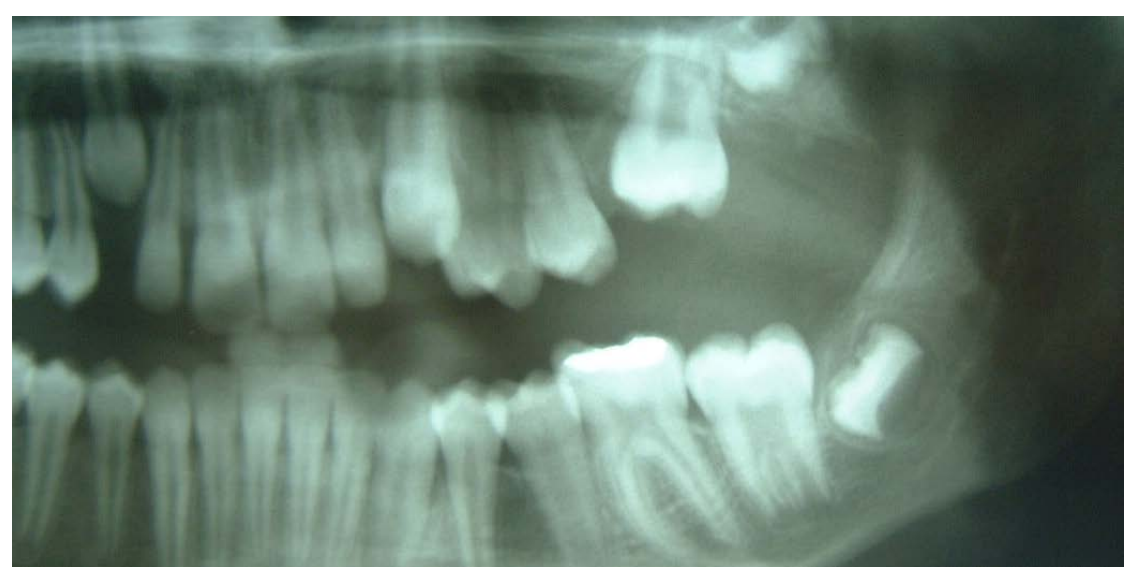

Figure 6. 8 months after the extraction of the left upper first molar.

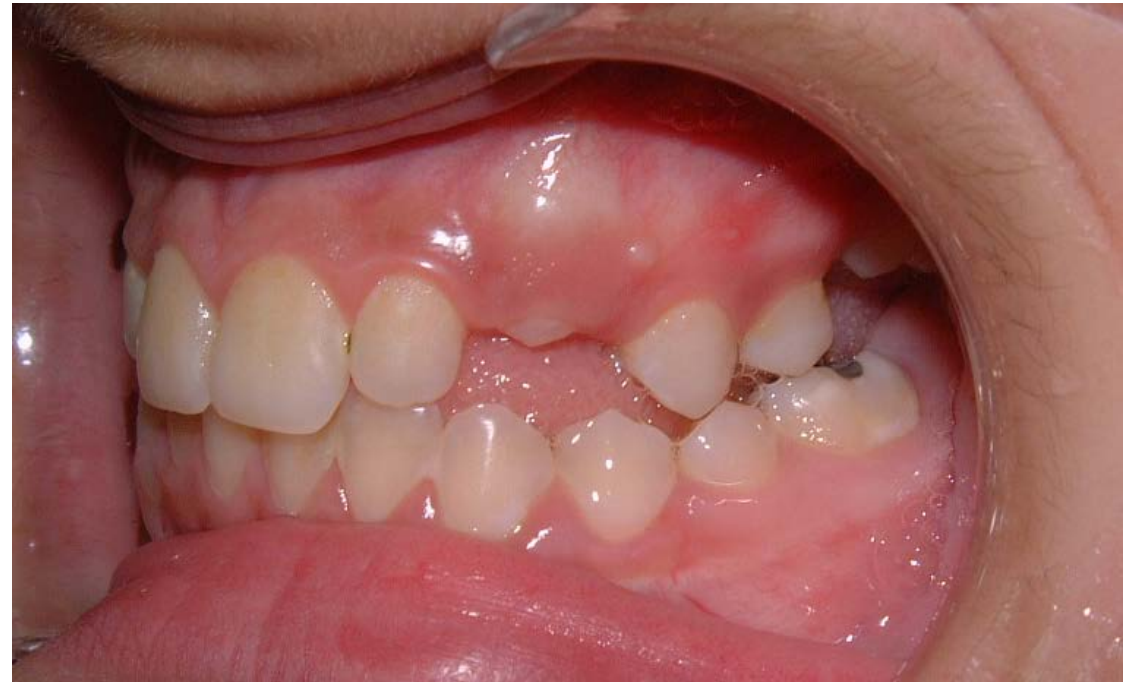

Figure 7. 12 months after the extraction of the upper left first molar, the second molar erupting distaly away from the second premolar. 
12 (23\%) USPM tooth erupted in slight cross bite (Figures 9-12).

\section{Discussion}

In many articles it is recommended to extract the heavily destructed UFPMs when the crowns of the USPMs are above the cementoenamel junction of the distobuccal root of the UFPMs to achieve a satisfactory closure of the extraction space by the mesial drift of the USPMs. In this research we tried to detect the best age for extraction of the heavily destructed upper first molars despite the eruption condition of the second permanent molars, although the eruption condition of many of the USPM teeth in this research were very near to the cementoenamel junction of the UFPMs [5] [9].

The extraction timing was planned to be at age 10.5 years old depending on tooth physiologic movement principles [10] [11]. The second permanent molar erupts in the normal conditions at age 12 years old, extracting the first permanent molar at age 10.5 will accelerate the eruption and the mesial drift of the second molar which will end in a good closure of the extraction space of the first molar without any orthodontic intervention. 84.6\% success was achieved and the USPM teeth erupted exactly at the distal contact of the upper second premolars. Our result is very near to the results of Jalevik \& Moller [9], 88\% of their patients did not need orthodontic treatment due to the extraction of the first molars.

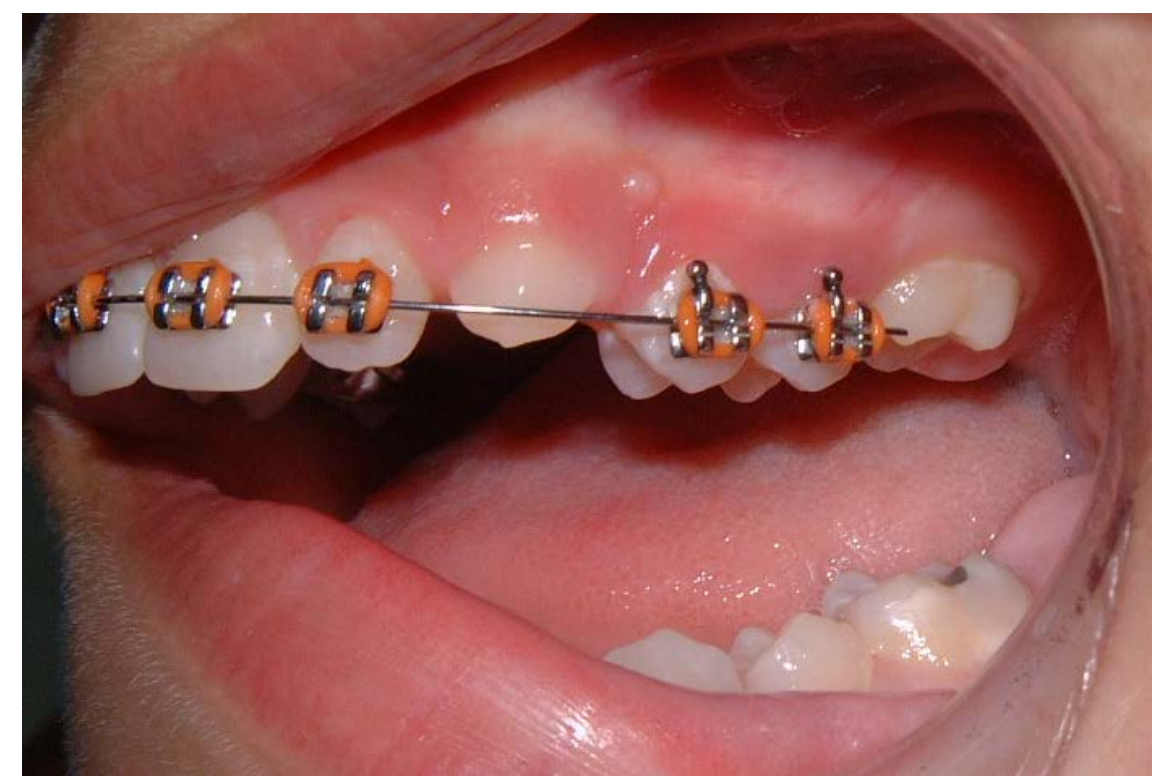

Figure 8. 17 months after the extraction, the second permanent molar erupted $1 \mathrm{~mm}$ distal to the second premolar.

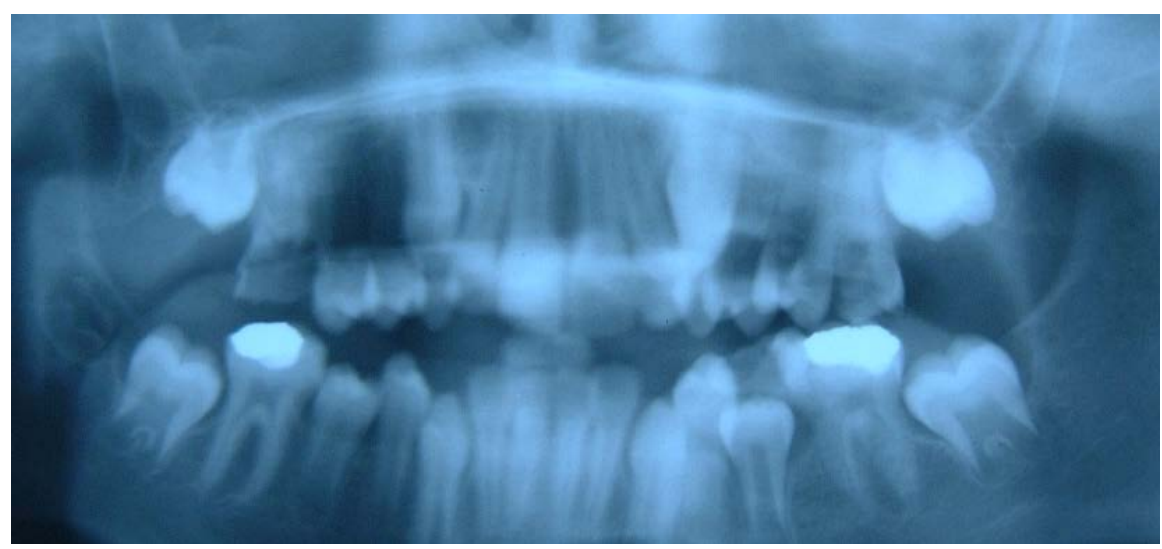

Figure 9. Exactly before the extraction of the left and right upper permanent first molars. 


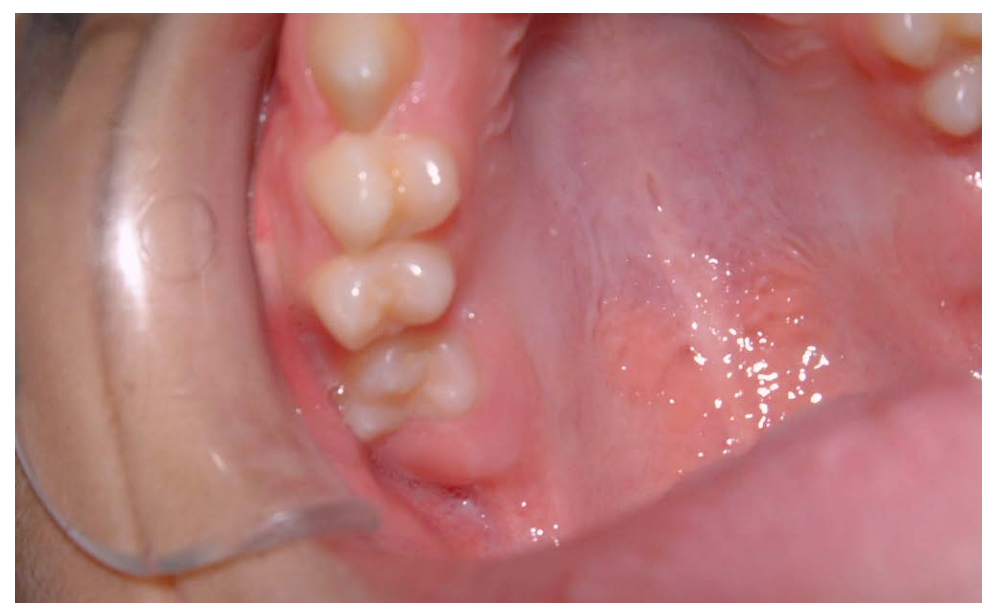

Figure 10. 14 months after extraction, the upper second permanent molar erupted distal to the second premolar but in cross bite, so it needed simple orthodontic treatment to be pushed buccaly.

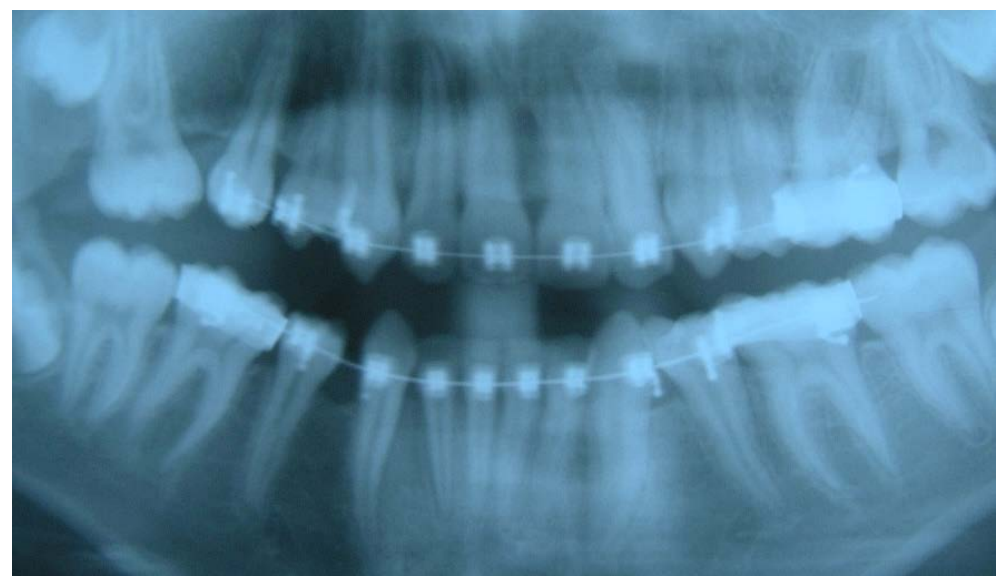

Figure 11. 18 months after extraction the upper molar erupted $1 \mathrm{~mm}$ distal and slightly in cross bite.

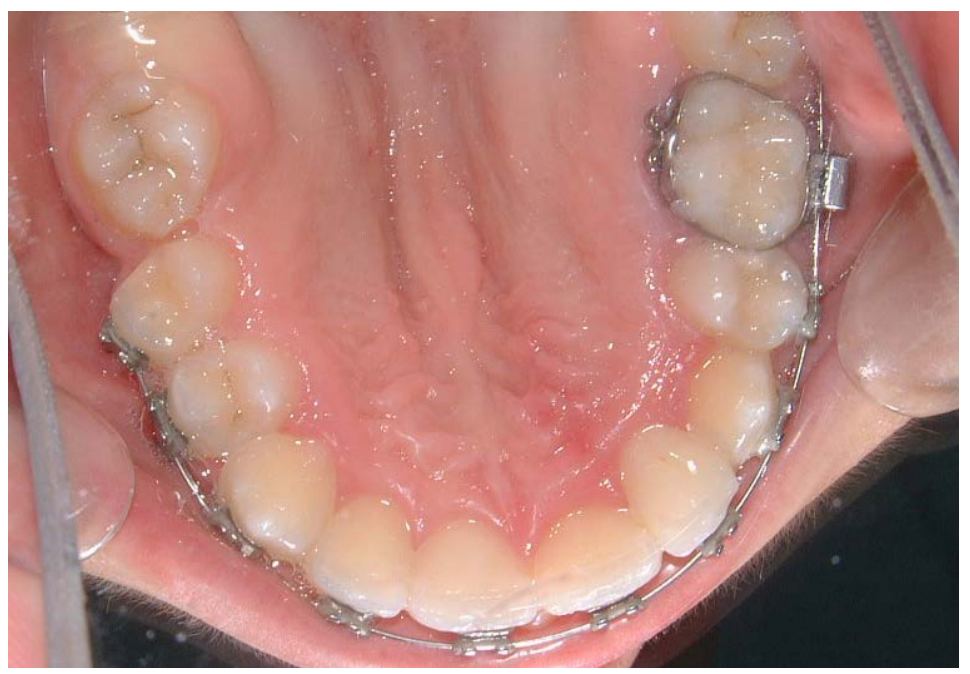

Figure 12. Intraoral photograph of the same patient, the distal eruption and the cross bite is very clear. 
All cases were Angle CLI molar relationship to prevent the over eruption of the lower first permanent molar. No lower molar over eruption was noticed in all cases.

$23 \%$ of the upper second molar teeth when they drift mesialy they rotate and tipped palataly, these cases needed simple orthodontic treatment to solve the cross bite of the erupted USPMs.

\section{Conclusion}

It must be emphasized that each individual case should be evaluated on its own merits, and not every case will be suited to extraction of FPMs. Despite this, extraction of compromised FPMs has the potential to provide significant advantages from the cost-benefit perspective in carefully selected cases. This treatment option does warrant serious consideration in any case where the long-term prognosis of such teeth is questionable, and especially if future fixed appliance orthodontic treatment is deemed to be desirable. If no or simple orthodontic treatment is required, and the third molar tooth is not missing, it is recommended to extract heavily destructed UFPMs at age of 10.5 years old in Angle CLI patients to achieve a complete closure of the extraction space by the passive mesial drift of the USPMs.

\section{Clinical Significance}

If a heavily destructed upper first permanent molar was extracted at age of 10.5 years old, most probably the extraction space will be completely closed by the passive mesial drift of the upper second permanent molar.

\section{References}

[1] Todd, J.E. and Dodd, T. (1983) Children’s Dental Health in the United Kingdom. Office of Population Censuses and Surveys, London.

[2] Seow, W.K. (1997) Clinical Diagnosis of Enamel Defects: Pitfalls and Practical Guidelines. International Journal of Dentistry, 47, 173-182. http://dx.doi.org/10.1002/j.1875-595X.1997.tb00783.x

[3] Sandler, P.J., Atkinson, R. and Murray, A.M. (2000) For Four Sixes. American Journal of Orthodontics and Dentofacial Orthopedics, 117, 418-434. http://dx.doi.org/10.1016/S0889-5406(00)70161-2

[4] Williams, J.K. and Gowans, A.J. (2003) Hypomineralised First Permanent Molars and the Orthodontist. European Journal of Paediatric Dentistry, 4, 129-132.

[5] Ong, D.-V. and Bleakley, J. (2010) Compromised First Permanent Molars: An Orthodontic Perspective. Australian Dental Journal, 55, 2-14. http://dx.doi.org/10.1111/j.1834-7819.2009.01176.x

[6] Plint, D.A. (1970) The Effect on the Occlusion of the Loss of One or More First Permanent Molars. Report of the Congress. European Orthodontic Society, 329-336.

[7] Williams, R. and Hosila, F.J. (1976) The Effect of Different Extraction Sites upon Incisor Retraction. American Journal of Orthodontics, 69, 388-410. http://dx.doi.org/10.1016/0002-9416(76)90208-6

[8] Gill, D.S., Lee, R.T. and Tredwin, C.J. (2001) Treatment Planning for the Loss of First Permanent Molars. Dental Update, 28, 304-308.

[9] Jalevik, B. and Moller, M. (2007) Evaluation of Spontaneous Space Closure and Development of Permanent Dentition after Extraction of Hypomineralized Permanent first Molars. International Journal of Paediatric Dentistry, 17, 328335. http://dx.doi.org/10.1111/j.1365-263X.2007.00849.x

[10] Hotz, R. (1970) Guidance of Eruption versus Serial Extraction. American Journal of Orthodontics, 58, 1-20. http://dx.doi.org/10.1016/0002-9416(70)90125-9

[11] Ulgen, M. (1983) Ortodontik Tedavi Prensipleri. Ankara Universitesi Dishekimligi Fakultesi Yayinlari, Ankara, 81-85. 